\title{
Causal interactions between the cerebral cortex and the autonomic nervous system
}

\author{
YU XiaoLin ${ }^{1},{\text { ZHANG } \text { Chong }^{1} \& \text { ZHANG JianBao }}^{2 *}$ \\ ${ }^{1}$ Department of Information Engineering, Officers College of Chinese People's Armed Police Force, Chengdu 610213, China; \\ ${ }^{2}$ Key Laboratory of Biomedical Information Engineering of Ministry of Education, Xi'an Jiaotong University, Xi'an 710049, China
}

Received August 20, 2013; accepted October 25, 2013; published online March 28, 2014

\begin{abstract}
Mental states such as stress and anxiety can cause heart disease. On the other hand, meditation can improve cardiac performance. In this study, the heart rate variability, directed transfer function and corrected conditional entropy were used to investigate the effects of mental tasks on cardiac performance, and the functional coupling between the cerebral cortex and the heart. When subjects tried to decrease their heart rate by volition, the sympathetic nervous system was inhibited and the heart rate decreased. When subjects tried to increase their heart rate by volition, the parasympathetic nervous system was inhibited and the sympathetic nervous system was stimulated, and the heart rate increased. When autonomic nervous system activity was regulated by mental tasks, the information flow from the post-central areas to the pre-central areas of the cerebral cortex increased, and there was greater coupling between the brain and the heart. Use of directed transfer function and corrected conditional entropy techniques enabled analysis of electroencephalographic recordings, and of the information flow causing functional coupling between the brain and the heart.
\end{abstract}

mental task, autonomic nervous system, cerebral cortex, heart rate variability, information flow

Citation: Yu XL, Zhang C, Zhang JB. Causal interactions between the cerebral cortex and the autonomic nervous system. Sci China Life Sci, 2014, 57: 532-538, doi: 10.1007/s11427-014-4627-0

Mental states such as stress and excitement can increase the risk of malignant arrhythmia and sudden cardiac death in patients with organic or ischemic heart disease. The interactions between the central and autonomic nervous systems have therefore attracted increasing attention. Complex interactions exist between the autonomic nervous system and various areas of the brain [1]. Stimulation of the cortex may activate the autonomic nervous system, resulting in changes in heart rate (HR) and blood pressure [2]. Different stresses may induce different patterns of autonomic activity $[3,4]$.

Extensive human and animal research has found that autonomic nervous system activity is influenced by various parts of the cerebral cortex, including the forebrain and post-central areas. For example, functional neuroimaging

*Corresponding author (email: zhangjb@mail.xjtu.edu.cn) and near-infrared spectroscopy studies found that the anterior cingulated cortex and prefrontal cortex were associated with stress-induced changes in sympathetic activity $[5,6]$. During lower body negative pressure experiments in which subjects were asked to perform decision-making tasks, the sympathetic skin response was associated with significant activity in the extrastriate visual cortical areas and parietal lobes, suggesting involvement of the post-central areas in the control of peripheral autonomic function [2,7]. However, the coupling patterns between the brain and the heart associated with sympathetic and parasympathetic activity have not been systematically studied.

The cortex plays a regulatory role in heart function, and receives feedback from the heart. Some researchers have used a combination of electroencephalographic (EEG) and electrocardiographic (ECG) recordings to guide decision 
making during surgical rehabilitation [8,9]. However, EEG and ECG recordings have only been used in isolation, and the relationships between these recordings have not been studied. A number of approaches have been proposed for the study of EEG activity, such as cross-correlation, spectral coherence and synchronization [10-12]. These techniques can show the connections among different brain areas, but cannot show the direction of information flow during functional coupling of EEG rhythms. In recent years, some researchers have used the directed transfer function (DTF) technique to show both the direction and strength of functional coupling. DTF has been widely applied to the analysis of neurophysiological signals, and has been used to estimate the direction of cortico-cortical and cortico- muscular information flow in experimental settings. The results were found to be consistent with the clinical diagnoses, and the technique was considered to be clinically effective and practical $[13,14]$. The DTF algorithm is based on the principle of causality, and initial analysis of two variables is subsequently extended to multiple variables. DTF is a linear analysis method based on the multivariate autoregressive (MVAR) model, and is useful for characterization of the interaction patterns among neurons in different states, and for determination of the directional impact of multivariate data between any two channels. DTF can provide a more rapid and direct reflection of brain functional coupling than conventional methods [15]. Faes et al. proposed the corrected conditional entropy (CCE) technique for the detection of nonlinear causality between dynamic systems [16]. This method has achieved better causal coupling values in visual-tactile cognitive magnetoencephalographic data analysis than DTF. Although numerous methods of EEG analysis are available, very few can analyze relationships between the brain and the heart. Most of these methods use statistical correlation analysis. In this study, we used DTF and CCE to investigate functional coupling in the cortex and directional features between the EEG and the RR interval of the ECG during volitional control of HR.

\section{Materials and methods}

\subsection{Subjects}

Fifty-four healthy male subjects, aged 22-27 years, voluntarily participated in the study. The subjects were asked to perform a task designed to decrease their HR by volition ( $\mathrm{HR}_{\text {slow }}$ task), followed by another task designed to increase their $\mathrm{HR}$ by volition $\left(\mathrm{HR}_{\text {fast }}\right.$ task). The subjects were instructed to avoid alcohol, tea, coffee and strenuous exercise for $12 \mathrm{~h}$ before the experiment, and they were carefully screened by obtaining a medical history and performing a physical examination. The study protocol was approved by the Xi' an Jiaotong University Ethics Committee, and written informed consent was obtained from each subject after the experimental protocol had been explained.

\subsection{Experimental protocol}

All experiments were performed between 7 and 10 p.m. Both the $\mathrm{HR}_{\text {slow }}$ and $\mathrm{HR}_{\text {fast }}$ tasks were performed in a quiet laboratory with the temperature controlled to $23 \pm 1^{\circ} \mathrm{C}$.

Baseline recordings were performed before the tasks were started. The RR intervals on the ECG were sent to a stimulus computer that produced auditory feedback sounds. The sounds corresponded to the HR, but were $10 \%$ slower during the $\mathrm{HR}_{\text {slow }}$ task and $10 \%$ faster during the $\mathrm{HR}_{\text {fast }}$ task (Figure 1A). The volitional command to decrease or increase the HR was guided by these sounds (Figure 1B).

The experimental protocol is shown in Figure 2. In the normal state, subjects simply relaxed and tried not to think of anything while sitting for $5 \mathrm{~min}$. During the baseline recordings, subjects listened to the sounds and subvocalized "one" with each sound, but did not think specifically about the heart and did not send a volitional command to the heart. During the $\mathrm{HR}_{\text {slow }}$ task, subjects first performed the baseline task for $5 \mathrm{~min}$, followed by $10 \mathrm{~min}$ of rest. They were then asked to concentrate on their heart and decrease their HR by volitional commands from the brain that were guided by the
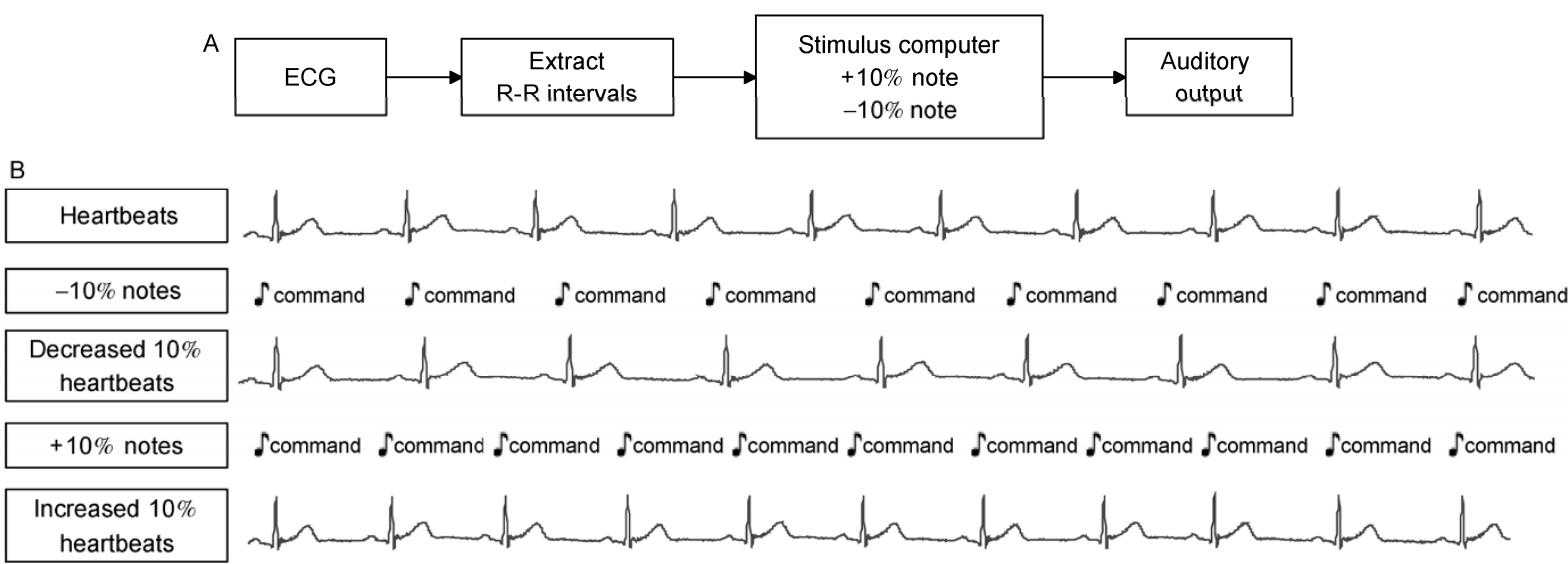

Figure 1 Experimental protocol. A, Experimental setup. B, The volitional command to decrease or increase the HR was guided by the sounds. 


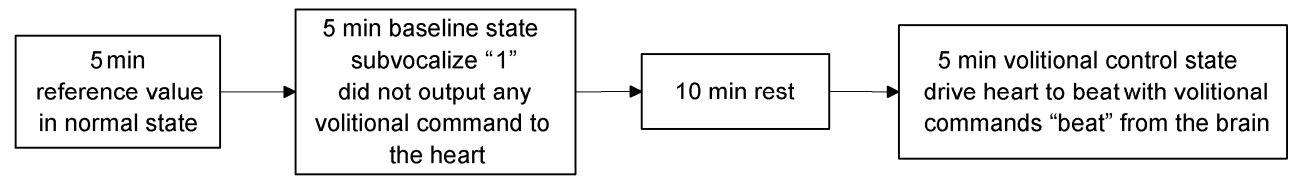

Figure 2 Experimental protocol.

slower "beat" of the sounds. During the baseline and volitional tasks, the EEG and ECG were recorded simultaneously. The $\mathrm{HR}_{\text {fast }}$ task followed the same protocol as the $\mathrm{HR}_{\text {slow }}$ task, except that the volitional commands were guided by faster sounds.

\subsection{Data acquisition}

The EEG was recorded using a Neuroscan 32 channel system (Neuroscan, USA). The cap electrodes were positioned according to the international 10-20 system, and the electrode impedances were below $5 \mathrm{k} \Omega$ per site. The EEG was recorded at 18 scalp locations: left and right frontal (FP1, FP2), mid-frontal (FC3, FC4 and FCz), central (C3, C4 and $\mathrm{Cz})$, parietal $(\mathrm{P} 3, \mathrm{P} 4$ and $\mathrm{Pz})$, occipital $(\mathrm{O} 1, \mathrm{O} 2$ and $\mathrm{Oz})$ and temporal (FT7, FT8, T7 and T8). All electrodes were referenced to linked ear lobe electrodes.

Surface $\mathrm{Ag} / \mathrm{AgCl}$ electrodes were attached to the chest to record the ECG (one electrode was placed over the right clavicle and one over the left ribs, and the grounding electrode was placed over the lower right ribs). Physiological signals were filtered by a band pass filter from 0.01 to $100 \mathrm{~Hz}$. The signal was sampled at $500 \mathrm{~Hz}$ and digitized by a 16-bit processor.

\subsection{Data analysis}

The algorithm based on wavelet transforms was used to obtain the RR intervals from the ECG. The HR was estimated from the RR intervals. Cardiac autonomic activity was assessed by power spectral analysis of the RR intervals, termed the heart rate variability (HRV). The magnitudes of the spectral frequencies of HRV are widely considered to reflect sympathetic and parasympathetic tone [17-19]. The spectral power of the HRV was calculated in the low frequency (LF: $0.04-0.15 \mathrm{~Hz}$ ) and high frequency (HF: $0.15-0.4 \mathrm{~Hz}$ ) ranges [20,21]. The fluctuation of HR in the LF range is influenced by the baroreceptor system, and reflects both sympathetic and parasympathetic activity [22]. The fluctuation of HR in the HF range is mainly related to parasympathetic efferent activity. The LF-to-HF ratio (LF/HF) reflects sympathetic outflow [23-25]. To minimize the effects of total power on the LF and HF components, these values were calculated in proportion to the total power in the frequency range from 0.04 to $0.4 \mathrm{~Hz}$, and the power was expressed in normalized LF (nuLF) and normalized HF (nuHF) units [24].

The DTF based on the MVAR model is useful for deter- mining the causal interaction patterns between neurons, and has been used to estimate the direction of cortico-cortical and cortico-muscular information flow in previous experiments [26]. According to the standardized rules published by Kaminski and Blinowska, the EEG data should be preliminarily normalized by subtracting the mean value and dividing by the variance before computing the DTF [27]. The MVAR model uses the following equation:

$$
\sum_{k=0}^{p} A(k) X(t-k)=E(t), \quad A(0)=I,
$$

where $X(t)=\left[x_{1}(t), x_{2}(t), \ldots, x_{N}(t)\right]^{\mathrm{T}}$ represents the $\mathrm{N}$ channel signals at time $t, E(t)=\left[e_{1}(t), e_{2}(t), \ldots, e_{N}(t)\right]^{\mathrm{T}}$ is a vector of zero-mean uncorrelated white noise process, $A(1), A(2), \ldots, A(p)$ are $N \times N$ matrices of model coefficients and $p$ is the model order.

To investigate the spectral properties of the examined process, the MVAR model is transformed to its frequency domain using the equations:

$$
\begin{gathered}
A(f) X(f)=E(f), \\
A(f)=\sum_{k=0}^{p} A(k) e^{-\mathrm{j} 2 \pi f \Delta t k},
\end{gathered}
$$

where $\Delta t$ is the temporal interval between two samples. The transformed MVAR model can then be rewritten as

$$
X(f)=A^{-1}(f) E(f)=H(f) E(f) .
$$

The DTF from the $j$ th to the $i$ th channel was defined as

$$
\gamma_{i j}^{2}(f)=\frac{\left|H_{i j}(f)\right|^{2}}{\sum_{m=1}^{N}\left|H_{i m}(f)\right|^{2}} .
$$

DTF is a normalized value ranging from zero to one. As the transfer function $H(f)$ is not a symmetric matrix, the difference between $\gamma_{i j}(f)$ and $\gamma_{j i}(f)$ reflected the direction of asymmetric information flow between the $j$ th and $i$ th channels. The EEG functional coupling of $\mathrm{D}_{\mathrm{FC} 3-\mathrm{Pz}}$ was recorded as the difference between the FC3-to- $\mathrm{Pz}$ and Pz-to-FC3 DTF values (FC3-to-Pz DTFdiff). A positive value indicates an FC3-to-Pz direction of functional cortical coupling, whereas a negative value indicates a Pz-to-FC3 direction of coupling. A value close to zero indicates a balanced directionality of functional cortical coupling. Functional cou- 
pling was recorded for other electrode pairs using the same method.

CCE based on embedding the multivariate time series is used to detect nonlinear causal interactions between dynamic systems. It has been successfully used for assessment of cardiovascular regulatory mechanisms, such as analysis of the variability in heart period, arterial pressure and respiratory rate; and investigation of the information flow across brain areas using multichannel scalp EEG recordings 28. First, a set of candidate components were defined as $S$. In the case of self embedding, $S=S_{Y}$; and in the case of mixed embedding, $S_{X} \cup S_{Y}$ 16. These values were defined as

$$
\begin{aligned}
& S_{Y}=\left\{y_{q}(n-l), q=1, \ldots, Q, l=1, \ldots, L_{\max }\right\}, \\
& S_{X}=\left\{x_{p}(n-l), p=1, \ldots, P, l=1, \ldots, L_{\max }\right\},
\end{aligned}
$$

Starting with an empty embedding vector $\boldsymbol{V}_{0}$, and computing the conditional entropy $C E\left(y_{j} \mid\left(z, V_{k-1}\right)\right)$, where $z$ is one of the elements of $S$; for each possible $z$, the candidate that minimizes $\mathrm{CE}$ is retained, to get $\arg \min \left\{C E\left(y_{j} \mid\left(z, V_{k-1}\right)\right)\right\}, \quad L_{\max }=k-1$.

The minimum CCE estimated as the output of the embedding procedure quantifies the unpredictability of the target series $y_{j}$ as the information carried by $y_{j}$ is not explained by the considered set of candidate components. This information is denoted as $I\left(y_{j} \mid S_{Y}\right)$ for self embedding, and as $I\left(y_{j} \mid S_{X} \cup S_{Y}\right)$ for mixed embedding. The information is then combined to quantify the causal coupling from $X$ to $Y$ using the formula:

$$
C_{X \rightarrow Y}=1-\frac{I\left(y_{j} \mid S_{X} \cup S_{Y}\right)}{I\left(y_{j} \mid S_{Y}\right)} .
$$

In this study, the model order was 7 , as estimated by the Akaike criterion suggested in previous DTF studies [26, 27]. EEG data from 18 electrodes were simultaneously entered into the MVAR model for computation of the directionality of the functional cortical coupling for all the pair combinations of these electrodes in the alpha and beta bands. The $\mathrm{RR}$ intervals and wavelet packet parameters of the EEG corresponding to each RR interval were used to compute the strength of coupling between the brain and the heart. The $\mathrm{RR}$ intervals and corresponding wavelet packet parameters were interpolated at one-second intervals by cubic spline interpolation, and the CCE was calculated. The results were expected to reflect the coupling strength from brain to heart and from heart to brain.

Results were analyzed with the paired $t$-test using SigmaPlot software. $P<0.05$ was considered statistically significant. Data are presented as mean \pm standard error of the mean.

\section{Results}

\subsection{HR and HRV}

During the $\mathrm{HR}_{\text {slow }}$ task, the HR, LF/HF and nuLF decreased significantly $(P<0.01)$, and $\mathrm{HF}$, nuHF and total power increased significantly $(P<0.01)$ compared with baseline values (Table 1). These results indicate that the HR can be decreased by volition, by decreasing sympathetic activity and increasing parasympathetic activity.

During the $\mathrm{HR}_{\text {fast }}$ task, the HR was significantly higher $\left(74 \pm 1.6\right.$ beats $\left.\mathrm{min}^{-1}\right)$ than at baseline $\left(71 \pm 1.5\right.$ beats $\left.\mathrm{min}^{-1}\right)$. The LF/HF and nuLF increased significantly, and HF and nuHF decreased significantly $(P<0.05)$ compared with baseline values (Table 2). These results show that sympathetic activity increased and parasympathetic activity decreased when the HR was increased by volition.

Table 1 Changes in HR and HRV during the $\mathrm{HR}_{\text {slow }}$ task ${ }^{\mathrm{a})}$

\begin{tabular}{cccccccc}
\hline & $\mathrm{HR}\left(\right.$ beats $\left.\mathrm{min}^{-1}\right)$ & $\mathrm{TP}\left(\mathrm{ms}^{2}\right)$ & $\mathrm{HF}\left(\mathrm{ms}^{2}\right)$ & $\mathrm{LF}\left(\mathrm{ms}^{2}\right)$ & $\mathrm{nuHF}$ & $\mathrm{nuLF}$ & $\mathrm{LF} / \mathrm{HF}$ \\
\hline Volition & $73.0 \pm 2.1^{* *}$ & $303.0 \pm 55.9^{*}$ & $146.0 \pm 32.0^{* *}$ & $144.0 \pm 24.7$ & $46.9 \pm 2.7^{* *}$ & $53.1 \pm 2.7^{* *}$ & $1.4 \pm 0.2^{* *}$ \\
Baseline & $75.0 \pm 2.2$ & $247.0 \pm 44.3$ & $106.0 \pm 27.5$ & $129.0 \pm 18.0$ & $37.7 \pm 3.1$ & $62.3 \pm 3.1$ & $2.1 \pm 0.3$ \\
\hline
\end{tabular}

a) Data are presented as mean \pm standard error of the mean. *, $P<0.05$; **, $P<0.01 \mathrm{vs.} \mathrm{baseline} \mathrm{(paired} t$-test). HRV, heart rate variability; HR, heart rate; TP, total power; HF, high frequency; LF, low frequency; nuHF, normalized high frequency; nuLF, normalized low frequency; LF/HF, LF-to-HF ratio.

Table 2 Changes in HR and HRV during the $\mathrm{HR}_{\text {fast }}$ task $^{\mathrm{a})}$

\begin{tabular}{cccccccc}
\hline & $\mathrm{HR}\left(\right.$ beats $\left.\mathrm{min}^{-1}\right)$ & $\mathrm{TP}\left(\mathrm{ms}^{2}\right)$ & $\mathrm{HF}\left(\mathrm{ms}^{2}\right)$ & $\mathrm{LF}\left(\mathrm{ms}^{2}\right)$ & $\mathrm{nuHF}$ & $\mathrm{nuLF}$ & $\mathrm{LF} / \mathrm{HF}$ \\
\hline Volition & $74.0 \pm 1.6^{* *}$ & $313.0 \pm 52.2$ & $98.0 \pm 21.4^{* * *}$ & $197.0 \pm 35.7$ & $31.2 \pm 2.3^{* *}$ & $68.8 \pm 2.3^{* *}$ & $2.8 \pm 0.3^{* * *}$ \\
Baseline & $71.0 \pm 1.5$ & $338.0 \pm 51.0$ & $154.0 \pm 25.2$ & $167.0 \pm 31.2$ & $47.4 \pm 3.4$ & $52.7 \pm 3.4$ & $1.5 \pm 0.2$ \\
\hline
\end{tabular}

a) Data are presented as mean \pm standard error of the mean. $*, P<0.05 ; * *, P<0.01$ vs. baseline (paired $t$-test). HRV, heart rate variability; HR, heart rate; TP, total power; HF, high frequency; LF, low frequency; nuHF, normalized high frequency; nuLF, normalized low frequency; LF/HF, LF-to-HF ratio. 


\subsection{Information flow among brain areas}

During the $\mathrm{HR}_{\text {slow }}$ task, the DTFdiff values from $\mathrm{Pz}$ to $\mathrm{FC} 3$, C3, C4, T7 and P3 and from P4 to C4 were all positive. These DTFdiff values all increased significantly in the alpha band compared with baseline values. In the beta band, the DTFdiff values from Pz to P3 and C4 increased significantly. During the $\mathrm{HR}_{\text {fast }}$ task, the DTFdiff values from $\mathrm{Pz}$ to FC3, FC4, C3, C4, T7, T8 and P3 and from P4 to FC3, FC4, $\mathrm{C} 3$ and $\mathrm{C} 4$ increased significantly in the alpha band compared with baseline values. The DTFdiff values from $\mathrm{Pz}$ to FC3, C4 and T7 and from P4 to FC3 and FC4 increased significantly in the beta band. These results show that the direction of information flow was predominantly from the parietal areas to the central and pre-central areas when the HR was controlled by volition (Tables 3 and $4 ; P<0.05$ ).

\subsection{Coupling strength between the brain and the heart}

During baseline and volitional control recordings, the coupling strength from brain to heart was significantly higher than the coupling strength from heart to brain (Figure 3). When the HR was decreased or increased by volition, the interactions between the brain and the heart tended to increase compared with baseline values, and there was a significant increase in coupling strength from $\mathrm{Pz}$ to the heart. These results indicate that the parietal areas were involved in processing volitional control and coupling between the brain and the heart.

\section{Discussion}

Autonomic nervous system activity affects the HR. Many non-pharmacological therapies used for treating cardiac arrhythmias affect the autonomic nervous system [29]. Qigong, yoga and acupuncture can change cardiac performance via autonomic nervous system pathways [30-33]. Learning and reward biofeedback mechanisms can be used to effectively treat cardiovascular diseases $[34,35]$. In this study, the HR was changed by volition. There was a significant decrease in $\mathrm{HR}$ during the $\mathrm{HR}_{\text {slow }}$ task and a significant increase in $\mathrm{HR}$ during the $\mathrm{HR}_{\text {fast }}$ task. The coupling strength from the parietal cortex to the heart was increased during these experiments. This may also be a useful method of changing autonomic nervous system activity in other related studies.

Although some researchers have suggested that all the frontal and post-central areas are associated with autonomic nervous system activity, the precise interactions between the anterior and posterior areas of the cerebral cortex that influence sympathetic and parasympathetic activity remain unclear. Anatomical studies indicate high connectivity be-

Table 3 DTFdiff values during the $\mathrm{HR}_{\text {slow }}$ task ${ }^{\mathrm{a})}$

\begin{tabular}{|c|c|c|c|c|}
\hline & $\alpha$-volition & $\alpha$-baseline & $\beta$-volition & $\beta$-baseline \\
\hline $\mathrm{D}_{\mathrm{FC} 3-\mathrm{Pz}}$ & $-0.19 \pm 0.02^{*}$ & $-0.16 \pm 0.02$ & $-0.06 \pm 0.02$ & $-0.06 \pm 0.02$ \\
\hline $\mathrm{D}_{\mathrm{C} 3-\mathrm{Pz}}$ & $-0.18 \pm 0.03^{* *}$ & $-0.15 \pm 0.03$ & $-0.08 \pm 0.02$ & $-0.07 \pm 0.02$ \\
\hline $\mathrm{D}_{\mathrm{C} 4-\mathrm{Pz}}$ & $-0.15 \pm 0.03^{*}$ & $-0.13 \pm 0.03$ & $-0.06 \pm 0.02^{*}$ & $-0.04 \pm 0.02$ \\
\hline $\mathrm{D}_{\mathrm{P} 3-\mathrm{Pz}}$ & $-0.17 \pm 0.03^{* *}$ & $-0.13 \pm 0.02$ & $-0.13 \pm 0.02^{*}$ & $-0.10 \pm 0.01$ \\
\hline $\mathrm{D}_{\mathrm{T} 7-\mathrm{Pz}}$ & $-0.23 \pm 0.02^{*}$ & $-0.19 \pm 0.02$ & $-0.11 \pm 0.02$ & $-0.10 \pm 0.02$ \\
\hline $\mathrm{D}_{\mathrm{C} 4-\mathrm{P} 4}$ & $-0.07 \pm 0.02^{*}$ & $-0.04 \pm 0.02$ & $0.00 \pm 0.02$ & $0.01 \pm 0.02$ \\
\hline
\end{tabular}

a) Data are presented as mean \pm standard error of the mean. ${ }^{*}, P<0.05 ; * *, P<0.01 \mathrm{vs}$. baseline (paired $t$-test).

Table 4 DTFdiff values during the $\mathrm{HR}_{\text {fast }} \operatorname{task}^{\mathrm{a})}$

\begin{tabular}{|c|c|c|c|c|}
\hline & $\alpha$-volition & $\alpha$-baseline & $\beta$-volition & $\beta$-baseline \\
\hline $\mathrm{D}_{\mathrm{FC} 3-\mathrm{Pz}}$ & $-0.20 \pm 0.02^{* *}$ & $-0.16 \pm 0.02$ & $-0.07 \pm 0.02^{*}$ & $-0.05 \pm 0.02$ \\
\hline $\mathrm{D}_{\mathrm{FC} 4-\mathrm{Pz}}$ & $-0.17 \pm 0.02^{*}$ & $-0.14 \pm 0.02$ & $-0.04 \pm 0.02$ & $-0.03 \pm 0.01$ \\
\hline $\mathrm{D}_{\mathrm{C} 3-\mathrm{Pz}}$ & $-0.20 \pm 0.03^{*}$ & $-0.15 \pm 0.02$ & $-0.10 \pm 0.02$ & $-0.08 \pm 0.02$ \\
\hline $\mathrm{D}_{\mathrm{C} 4-\mathrm{Pz}}$ & $-0.16 \pm 0.03^{*}$ & $-0.13 \pm 0.02$ & $-0.06 \pm 0.02^{*}$ & $-0.04 \pm 0.01$ \\
\hline $\mathrm{D}_{\mathrm{P} 3-\mathrm{Pz}}$ & $-0.18 \pm 0.02^{* *}$ & $-0.13 \pm 0.03$ & $-0.13 \pm 0.02$ & $-0.12 \pm 0.01$ \\
\hline $\mathrm{D}_{\mathrm{T} 7-\mathrm{Pz}}$ & $-0.25 \pm 0.02^{*}$ & $-0.21 \pm 0.02$ & $-0.14 \pm 0.02^{*}$ & $-0.11 \pm 0.02$ \\
\hline $\mathrm{D}_{\mathrm{FC} 3-\mathrm{P} 4}$ & $-0.20 \pm 0.02^{*}$ & $-0.16 \pm 0.02$ & $-0.05 \pm 0.02^{*}$ & $-0.02 \pm 0.01$ \\
\hline $\mathrm{D}_{\mathrm{FC} 4-\mathrm{P} 4}$ & $-0.14 \pm 0.02^{* *}$ & $-0.11 \pm 0.02$ & $-0.01 \pm 0.01^{*}$ & $0.01 \pm 0.01$ \\
\hline $\mathrm{D}_{\mathrm{C3}-\mathrm{P} 4}$ & $-0.13 \pm 0.02^{*}$ & $-0.10 \pm 0.02$ & $-0.03 \pm 0.01$ & $-0.02 \pm 0.01$ \\
\hline $\mathrm{D}_{\mathrm{C} 4-\mathrm{P} 4}$ & $-0.07 \pm 0.03^{*}$ & $-0.04 \pm 0.02$ & $0.01 \pm 0.02$ & $0.01 \pm 0.02$ \\
\hline
\end{tabular}

a) Data are presented as mean \pm standard error of the mean. ${ }^{*}, P<0.05 ; * *, P<0.01$ vs. baseline (paired $t$-test). 

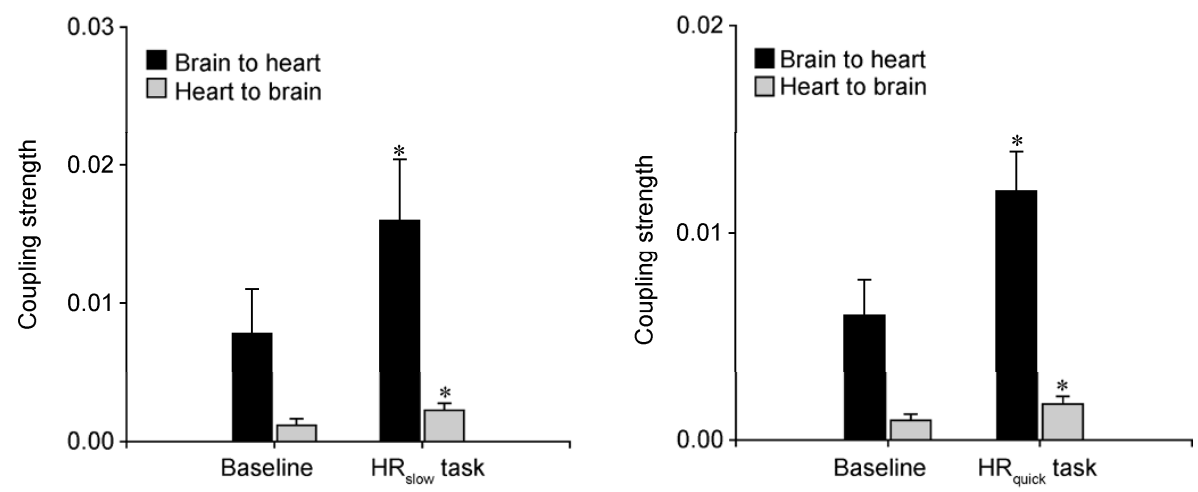

Figure 3 Causal coupling between the RR intervals and the EEG during volitional control of HR. *, $P<0.05$ (paired $t$-test).

tween the frontal and posterior areas of the cerebral cortex [36]. In this study, the DTF method was used to investigate the functional relationships between different areas of the cortex. Our results showed increased flow of information from the parietal areas to the pre-central areas during the experiments. The parietal areas were the "source" locations of information outflow during the volitional control tasks. These findings indicate that during the volitional control of $\mathrm{HR}$, the dominant direction of information flow was from the parietal areas to the sensorimotor cortex, suggesting that the parietal areas play an important role in triggering the sensorimotor cortex and integrating information.

The brain usually regulates cardiovascular function via the autonomic nervous system and the endocrine system. Interactions between the brain and the heart regulate cardiac activity and vasomotor function in response to stimuli such as strenuous exercise, panic, environmental stress and emotional changes. Many studies have investigated the brain areas that may influence cardiovascular function, and the feedback from the heart to the brain [2,7]. However, the functional coupling patterns between brain and heart activity have not been adequately studied. To investigate the functional relevance of coupling between the brain and the heart, CCE based on embedding the multivariate time series was used to detect nonlinear causal interactions between the brain and the heart. This method has been successfully used for assessment of cardiovascular regulatory mechanisms, such as analysis of the variability in heart period, arterial pressure, and respiratory rate; and investigation of the information flow across brain areas [28]. ECG data cannot be used in isolation to investigate the information flow between the brain and the heart, because the autonomic nervous system regulates cardiac performance by changing the RR interval. EEG activity was reported to be synchronized to the cardiac cycle and fluctuations in HRV [37]. Our preliminary findings showed correlation between the wavelet packet power of the EEG and the HRV $[38,39]$. We therefore used the wavelet packet power of the EEG and the RR intervals to demonstrate connectivity between the brain and the heart in this study. Our results show that the cou- pling strengths from brain to heart and from heart to brain were both increased during the volitional control tasks. Information flow from the "source" location to the heart increased significantly. The coupling strength was greater from brain to heart than from heart to brain at baseline as well as during the volitional control tasks. Using the partial directed coherence method, Abukonna et al. found that the information flow from the parietal area to the heart increased during volitional control [38]. Performance of mental arithmetic was also found to increased the coupling strength between the parietal area and the heart [39]. Our results indicate that volition changes the coupling strength, and can decrease or increase the HR by changing sympathetic and parasympathetic activity. The DTF and CCE methods are expected to become the index methods for detecting functional coupling between different brain areas, and information flow between the brain and the heart. Several functional brain imaging studies demonstrated gender differences in regional brain activity during different cognitive tasks [40]. Therefore, all the subjects in this study were healthy males. Further studies, using a wider range of subjects including females, are necessary to confirm our conclusions.

The work was supported by the National Natural Science Foundation of China (61302011).

1 Verberne AM, Owens NC. Cortical modulation of the cardiovascular system. Prog Neurobiol, 1998, 154: 149-168

2 Critchley HD, Corfield DR, Chandler MP, Mathias CJ, Dolan RJ. Cerebral correlates of autonomic cardiovascular arousal: a functional neuroimaging investigation in humans. J Physiol, 2000, 523: 259-270

3 Santaella DF, Devesa CR, Rojo MR, Amato MB, Drager LF, Casali KR, Montano N, Lorenzi-Filho G. Yoga respiratory training improves respiratory function and cardiac sympathovagal balance in elderly subjects: a randomised controlled trial. BMJ Open, 2011, 1: e000085

4 Dabhade AM, Pawar BH, Ghunage MS, Ghunage VM. Effect of pranayama (breathing exercise) on arrhythmias in the human heart. Explore (NY), 2012, 8: 12-15

5 Nugent AC, Bain EE, Thayer JF, Sollers JJ, Drevets WC. Sex differences in the neural correlates of autonomic arousal: a pilot PET study. 
Int J Psychophysiol, 2011, 80: 182-191

6 Yasui H, Takamoto K, Hori E, Urakawa S, Nagashima Y, Yada Y, Ono T, Nishijo H. Significant correlation between autonomic nervous activity and cerebral hemodynamics during thermotherapy on the neck. Autonom Neurosci, 2010, 156: 96-103

7 Kimmerly DS, O'Leary DD, Menon RS, Gati JS, Shoemaker JK. Cortical regions associated with autonomic cardiovascular regulation during lower body negative pressure in humans. J Physiol, 2005, 569: 331-345

8 Lay-Ekuakille A, Vergallo P, Trabacca A, De Rinaldis M, Angelillo F, Conversano F, Casciaro S. Low-frequency detection in ECG signals and joint EEG-Ergospirometric measurements for precautionary diagnosis. Measurement, 2013, 46: 97-107

9 Cuevas K, Bell MA. EEG and ECG from 5 to 10 months of age: developmental changes in baseline activation and cognitive processing during a working memory task. Int J Psychophysiol, 2011, 80: 119-128

10 Murata T, Takahashi T, Hamada T, Omori M, Kosaka H, Yoshida H, Wada Y. Individual trait anxiety levels charactering the properties of zen meditation. Neuropsychobiology, 2004, 50: 189-194

11 Stam CJ, Van Dijk BW. Synchronization likelihood: an unbiased measure of generalized synchronization in multivariate data sets, Physica D, 2002, 163: 236-251

12 Urbano A, Babiloni C, Onorati P, Babiloni F. Dynamic functional coupling of high resolution EEG potentials related to unilateral internally triggered one-digit movements. Electroencephalogr Clin Neurophysiol, 1998, 106: 477-487

13 Vecchio F, Babiloni C, Buffo P, Rossini PM, Bertini M. Inter-hemispherical functional coupling of EEG rhythms during the perception of facial emotional expressions. Clin Neurophysiol, 2013, 124: 263-272

14 Florin E, Gross J, Pfeifer J, Fink GR, Timmermann L. Reliability of multivariate causality measures for neural data. J Neurosci Methods, 2011, 198: 344-358

15 Pereda E, Quiroga RQ, Bhattacharya J. Nonlinear multivariate analysis of neurophysiological signals. Prog Neurobiol, 2005, 77: 1-37

16 Faes L, Nollo G, Erla S, Papadelis C, Braun C, Porta A. Detecting nonlinear causal interactions between dynamical systems by non-uniform embedding of multiple time series. Conf Proc IEEE Eng Med Biol Soc, 2010, 32: 102-105

17 Brunner EJ, Hemingway H, Walker BR, Page M, Clarke P, Juneja M, Shipley MJ, Kumari M, Andrew R, Seckl JR, Papadopoulos A, Checkley S, Rumley A, Lowe GD, Stansfeld SA, Marmot MG. Adrenocortical, autonomic, and inflammatory causes of the metabolic syndrome: nested case-control study. Circulation, 2002, 106: 2659-2665

18 Salomé N, Ngampramuan S, Nalivaiko E. Intra-amygdala injection of GABAA agonist, muscimol, reduces tachycardia and modifies cardiac sympatho-vagal balance during restraint stress in rats. Neuroscience, 2007, 148: 335-341

19 Stein PK, Bosner MS, Kleiger RE, Conger BM. Heart rate variability: a measure of cardiac autonomic tone. Am Heart J, 1994, 127: $1376-1381$

20 Camm AJ, Malik M, Bigger JT, Breithardt G, Cerutti S, Cohen RJ, Coumel P, Fallen EL, Kennedy HL, Kleiger RE, Lombardi F, Malliani A, Moss AJ, Rottman JN, Schmidt G, Schwartz PJ, Singer DH. Heart rate variability: standards of measurement, physiological interpretation and clinical use. Task Force of the European Society of Cardiology and the North American Society of Pacing and Electro- physiology. Circulation, 1996, 93: 1043-1065

21 Burr RL, Cowan MJ. Autoregressive spectral models of heart rate variability. J Electrocardiol, 1992, 25: 224-233

22 Hilz MJ, Devinsky O, Szczepanska H, Borod JC, Marthol H, Tutaj M. Right ventromedial prefrontal lesions result in paradoxical cardiovascular activation with emotional stimuli. Brain, 2006, 129: 3343-3355

23 Lumbers ER, Yan YZ. A method for determining baroreflex mediated sympathetic and parasympathetic control of the heart in pregnant and non-pregnant sheep. J Physiol, 1999, 515: 555-566

24 Malliani A, Lombardi F, Pagani M, Cerutti S. Power spectral analysis of cardiovascular variability in patients at risk for sudden cardiac death. J Cardiovasc Electrophysiol, 1994, 5: 274-286

25 Mateo J, Laguna P. Improved heart rate variability signal analysis from the beat occurrence times according to the IPFM model. IEEE Trans Biomed Eng, 2000, 47: 985-996

26 De Gennaro L, Vecchio F, Ferrara M, Curcio G, Rossini PM, Babiloni C. Antero-posterior functional coupling at sleep onset: changes as a function of increased sleep pressure. Brain Res Bull, 2005, 65: 133-140

27 Kaminski MJ, Blinowska KJ. A new method of the description of the information flow in the brain structures. Biol Cybern, 1991, 65: 203-210

28 Faes L, Nollo G, Porta A. Information-based detection of nonlinear Granger causality in multivariate processes via a nonuniform embedding technique. Phys Rev, 2011, 83: 051112

29 Nattel S. New ideas about atrial fibrillation 50 years on. Nature, 2002, 415: $219-226$

30 Jayasinghe SR. Yoga in cardiac health (a review). Eur J Cardiovasc Prev Rehabil, 2004, 11: 369-375

31 Sakai S, Hori E, Umeno K, Kitabayashi N, Ono T, Nishijo H. Specific acupuncture sensation correlates with EEGs and autonomic changes in human subjects. Auton Neurosci-Basic, 2007, 133: 158-169

32 Shannahoff-Khalsa DS, Sramek BB, Kennel MB, Jamieson SW. Hemodynamic observations on a yogic breathing technique claimed to help eliminate and prevent heart attacks: a pilot study. J Altern Complement Med, 2004, 10: 757-766

33 Skoglund L, Jansson E. Qigong reduces stress in computer operators. Compl Ther Clin Pract, 2007, 13: 78-84

34 Nakao M, Yano E, Nomura S, Kuboki T. Blood pressure-lowering effects of biofeedback treatment in hypertension: a meta-analysis of randomized controlled trials. Hypertens Res, 2003, 26: 37-45

35 Schwartz GE. Voluntary control of human cardiovascular integration and differentiation through feedback and reward. Science, 1972, 175: 90-93

36 Goldman-Rakic PS. Topography of cognition: parallel distributed networks in primate association cortex. Annu Rev Neurosci, 1998, 11: $137-156$

37 McCraty R. Influence of cardiac afferent input on heart-brain synchronization and cognitive performance. Int J Psychophysiol, 2002, 45: 72-73

38 Abukonna A, Yu X, Zhang C, Zhang J. Volitional control of the heart rate. Int J Psychophysiol, 2013

39 Yu XL, Zhang JB, Xie DD, Wang J, Zhang C. Relationship between scalp potential and autonomic nervous activity during a mental arithmetic task. Autonom Neurosci, 2009, 146: 81-86

40 Hamann S, Canli T. Individual differences in emotion processing. Curr Opin Neurobiol, 2004, 14: 233-238

Open Access This article is distributed under the terms of the Creative Commons Attribution License which permits any use, distribution, and reproduction in any medium, provided the original author(s) and source are credited. 